\title{
Using a Machine Learning Algorithm to Create a Computational Artwork: Variable
}

\begin{abstract}
Selçuk Artut ${ }^{*}$
As computational systems have become an integral part of our daily lives, we often see that contemporary art also has adapted itself with the newborn technological changes in diversified dimensions. Machine Learning, which has recently become a remarkable development in science, has also begun to manifest itself in various artistic works. As accordingly, the artwork that has been created by the author of this article named "Variable" stands as an interactive work of art that embraces machine learning algorithms within its compositional structure. The artwork was extensively influenced by the sophisticated discourse of German philosopher Heidegger's book "Being and Time." Consequently, Being and Time text has been taught to a machine learning system, and thus the system has been able to automatically generate new original contents when the viewer interacts with the touch of a button. The generative system performs its Machine Learning Markov Chain operations with the implementation of a Python programming language-based library named Markovify. The work constantly redefines its own artistic title and statement with the use of a machine learning framework. In this article, the contribution of machine learning to the production of artworks is being examined while focusing on various implementations.
\end{abstract}

\section{Introduction}

Machine Learning has received a notable amount of attraction in the past few decades. With the introduction of Bayes Theorem, the underpinnings of the concept may refer to the early $19^{\text {th }}$ century, but its framework has been progressively developed and substantial accomplishments have been achieved over the course of couple years. Machine learning aspires to mimic human intelligence while gathering data from previous experience and suits them in a reasonable structure with associated outcomes. Unlike the common sense of computers as being a well-defined deterministic system, machine learning uses the given set of data to interpret statistical reasoning with no explicit design of a deterministic system. Machine learning extensively applies computational methods to perform statistical operations that puts suitable models for interpreting explicit results. With the employment of computational statistics, Computer Science has been hosting the science of machine learning to formulate new findings in various fields of applications. Some of the popular applications of use would be in Bioinformatics, Computer Vision, Economics, Insurance,

\footnotetext{
*Assistant Professor, Visual Arts and Visual Communication Design, Sabanci University, Turkey.
} 
Search Engine Engineering, Financial Market Analysis etc. ${ }^{1}$ Correspondingly, machine learning has a far-reaching potential to be investigated with diversified implications in solving complex problems. As an example, medical scientists have been investigating how to use machine learning for early detection of terminal diseases such as cancer and aids. The researchers imply that the machine learning is helping to improve basic understanding of cancer development and progression. ${ }^{2}$ Additionally, various researchers have been analyzing stock market predictions using machine learning paradigms. ${ }^{3}$

According to Arthur Samuel, ${ }^{4}$ machine learning is a field of computer science that gives computers the ability to learn without being explicitly programmed. Géron ${ }^{5}$ defines machine learning as the science and the art of programming computers so they can learn from data. Mitchell ${ }^{6}$ defines machine learning as a field that examines how computer programming can be set up so that computers can evolve automatically from experience. To formulate algorithms based on exploratory data analysis, researchers have been implementing various methods of data sciences. Previously symbolic/ knowledge-based systems have been accepted as more capable of machine learning systems. However, with the recent abundance of data, more research was studied to generate experience-based systems for getting effective results.

1. A. Waibel, T. Hanazawa, G. Hinton, K. Shikano, and K. J. Lang, Phoneme recognition using time-delay neural networks. IEEE Transactions on Acoustics, Speech, and Signal Processing 37, no. 3, (1989); D. Pomerleau, and T. Jochem, Rapidly adapting machine vision for automated vehicle steering. IEEE Expert 11, no. 2 (1996); G. F. Cooper, C. F. Aliferis, R. Ambrosino, J. Aronis, B. G. Buchanan, R. Caruana, et al., An evaluation of machine-learning methods for predicting pneumonia mortality. Artificial Intelligence in Medicine 9, no. 2 (1997).

2. Joseph A. Cruz, and David S. Wishart, Applications of machine learning in cancer prediction and prognosis. Cancer Informatics 2 (2006); Sung-Bae Cho, and Hong-Hee Won, Machine learning in DNA microarray analysis for cancer classification. In Proceedings of the First Asia-Pacific bioinformatics conference on Bioinformatics 19 (2003).

3. Robert P. Schumaker, and Hsinchun Chen, Textual analysis of stock market prediction using breaking financial news: The AZFin text system. ACM Transactions on Information Systems (TOIS) 27, no. 2 (2009), 12; Paul D. Yoo, Maria H. Kim, and Tony Jan, Machine learning techniques and use of event information for stock market prediction: A survey and evaluation. International Conference on Computational Intelligence for Modeling, Control and Automation (CIMCA 2005) (835-841) (Piscataway, NJ: IEEE, 2005); Jigar Patel, Sahil Shah, Priyank Thakkar, and K. Kotecha, Predicting stock market index using fusion of machine learning techniques. Expert Systems with Applications 42, no. 4 (2015).

4. Arthur L. Samuel, Some studies in machine learning using the game of checkers. IBM Journal of Research and Development 3, no. 3 (1959).

5. Aurélien Géron, Hands-On Machine Learning With Scikit-Learn and Tensor Flow: Concepts, Tools, and Techniques to Build Intelligent Systems (Sebastopol, CA: O'Reilly Media, 2017), 4.

6. Tom M. Mitchell, Machine Learning (New York: McGraw-Hill, 1997). 
As humans, we frequently address learning to enhance our thinking to harvest knowledge. Nevertheless, humans generally learn things unconsciously and as a result, it becomes quite difficult to simulate the structure of a human intelligence deeply. Due to the increasing demand from related fields of study, researchers in this field are extensively working on building efficient algorithms to digest statistical data with machine learning structures. However, it is not only the science applications benefitting from implementations of machine learning. Progressively artists are starting to seek how machine learning can be employed in the process of art-making. Consequently, machine learning can be used to drive new and compelling ideas for the arts community as equally as well.

\section{Related Work}

Computational systems have become an integral part of our contemporary lives and arts also has been deeply influenced by the recent technological advancements in machine learning. As a consequence, there is a growing number of artists who are ambitious to create new forms of art with the use of machine learning. As a composer of interactive electronic music, Laetitia Sonami is best known for her live-performances with the use of an electronic apparatus named Lady's Glove. Lady's glove consists of various electronic sensors and actuators that are used to manipulate sound elements located on a connected computer. In the same manner, Sonami later created the Lady's Glove' s successor the Spring Spyre with a different computational structure. Spring Spyre employs a machine learning tool named Wekinator that is a free, opensource software developed by a researcher named Rebecca Fiebrink. According to Fiebrink, ${ }^{7}$ Wekinator lets users create new musical systems with data provided by gestural components.

Sonami, in 2016, defines her experience as a gained flexibility with the fun of exploring exciting results. Previously with the Lady's Glove, her performances have relied on explicit rules defined apriori by the artists' decisions in building an interactive system. Therefore she needed to apply certain filters to separate various types of gestures from the each other. Later with the addition of machine learning, Sonami was allowed to reverse the decision-making orientation by teaching performative gestures to the system and after recalling them during her live performances (Figure 1).

7. Rebecca Fiebrink, Machine Learning as Meta-Instrument: Human-Machine Partnerships Shaping Expressive Instrumental Creation. In Musical Instruments in the 21st Century, ed. Till Bovermann, Alberto de Campo, Hauke Egermann, Sarah-Indriyati Hardjowirogo, and Stefan Weinzierl (137-151) (Singapore: Springer, 2017). 
Figure 1. Spring Spyre by Laetitia Sonami

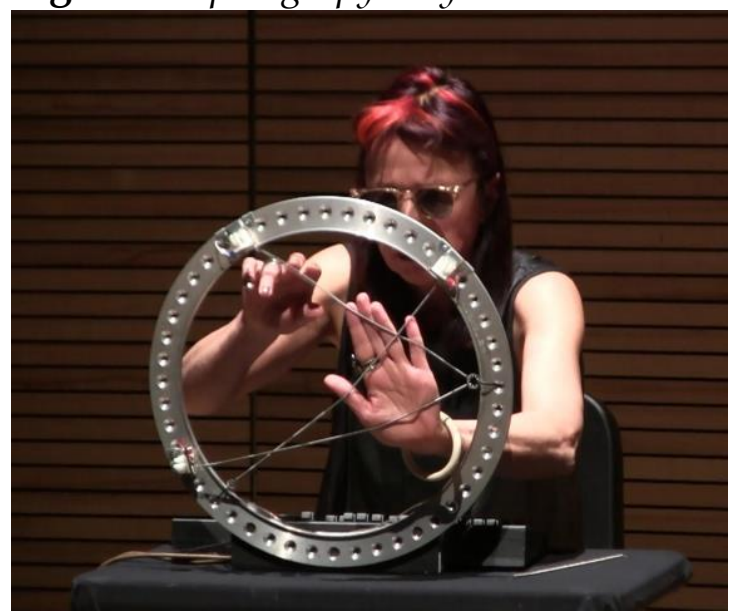

Similar to Sonami, Gene Kogan is an artist working with programming skills to find cross sections of computer science with arts. Kogan has been working on developing a free educational resource "ml4a" for artists who would want to integrate machine learning into their art projects. In addition to his academic activities, Kogan creates distinctive works of art with extensive machine learning influence. Kogan's artwork titled Doodle Tunes ${ }^{8}$ enables the users to turn simple hand drawings of musical instruments into computer-based sound sample players (Figure 2).

Figure 2. Doodle Tunes by Gene Kogan

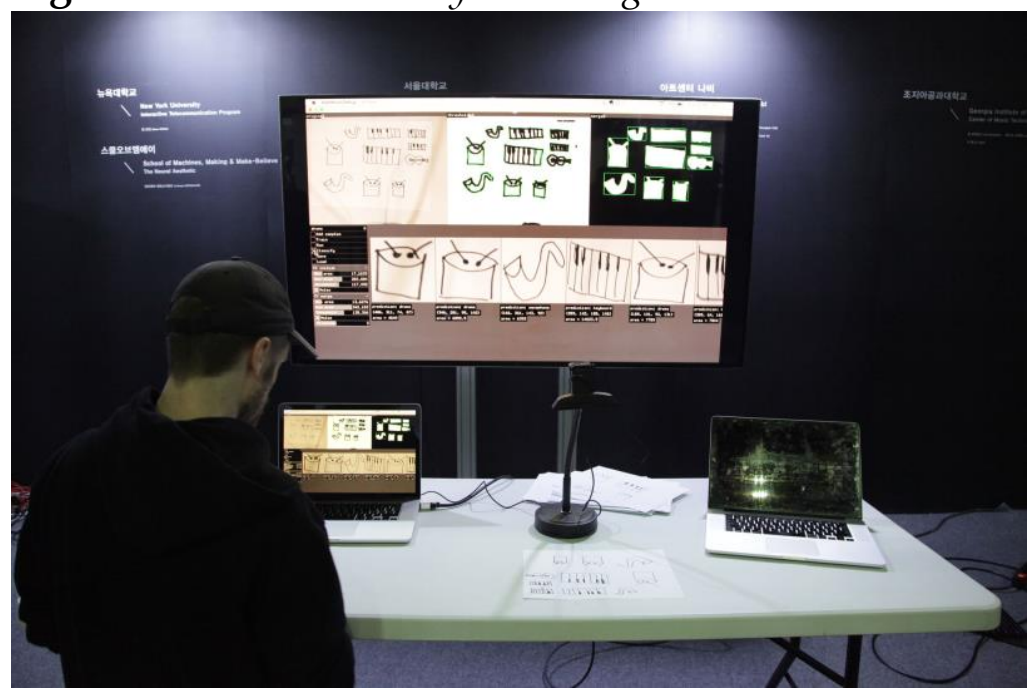

Kogan implements convolutional neural networks to analyze the handdrawings with computer vision techniques and classifies them into musical

8. Doodle Tunes. Retrieved from https://bit.ly/2zHllra. 
instruments with certain prediction values. In another work titled Neural Synthesis, in 2017, Kogan experiments with a technique called neural synthesis. According to Kogan, neural synthesis manipulates bitmap images with a pixelbased optimization technique to attain some desired level of activations in a convolutional neural network. In his video artwork submitted to an Annual Conference on Neural Information Processing Systems, ${ }^{9}$ it is observed that the dream-like hallucinogenic visual content reminds us the similar processing technique that was used in the famous artwork "Deepdream" by Alexander Mordvintsev. Similarly, we are observing a certain kind of a visual abstraction with the use of the neural synthesis technique. In Deep Dream example, Mordvintsev et al. ${ }^{10}$ have trained an artificial neural network with millions of training data to adjust the system to deliver the desired set of classification schemes. Based on this classification scheme, researchers then require the system to give them a qualitative sense of the level of abstraction which they name "Inceptionism." As a result, the machine learning enables the researcher to deliver unexpected visual contents within an iterative process (Figure 3).

Figure 3. Deep Dream

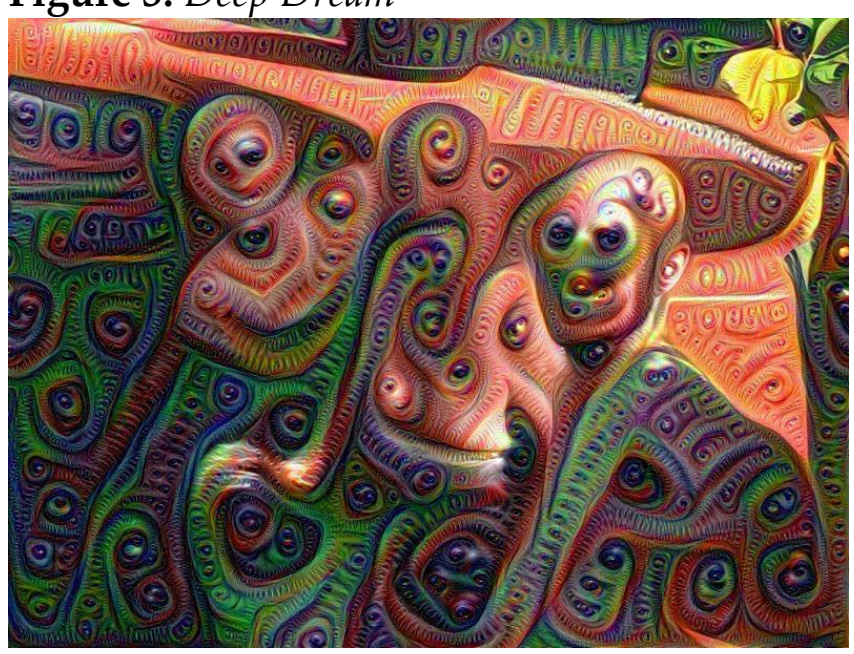

Likewise, Markov Chains have been implemented in a project called The Mansfield Reporter by Max Lupo. ${ }^{11}$ According to Lupo, The Mansfield Reporter is a simple device that creates new texts generated from some of history's greatest authors like Friedrich Nietzsche, William Shakespeare, or Gertrude Stein etc. Based on the previous corpus of texts, the algorithm uses statistical methods to create similar texts that have a structural resemblance to well-known authors

9. Neural synthesis. Retrieved from https://bit.ly/2DvzQ5c.

10. Alexander Mordvintsev, Christopher Olah, and Mike Tyka, Inceptionism: Going deeper into neural networks (June 17, 2015). Retrieved from https://bit.ly/2M5NfAg.

11. Max Lupo, The Mansfield Reporter (November 1, 2015). Retrieved from https://bit.ly/2OWoRD8. 
of the past. With a substantial amount of previous data, Markov Chains enable us to stochastically forecast probabilistic models with a reliable outcome.

\section{Methodology}

The work presented in this paper was manifested as a piece of interactive art, taking the form of an installation within a group show at the Futuretellers Exhibition, Zorlu PSM Istanbul in 2017. This piece is built on establishing an environment that utilizes Machine Learning algorithms to generate artistic discourse based on multiple computational systems. Because of the distributed computational necessities required by the art piece, the idea of having a hub of interconnected computers have been pursued to handle the results of the machine learning algorithms. In particular, nine micro-size computers (Raspberry) were interconnected to a local network and the communication traffic was accomplished with the use of a network protocol named Open Sound Control (OSC). ${ }^{12}$ OSC is a networking protocol developed to estanblish data communications between a variety of multimedia devices optimized for computers. Originally when the protocol was created, its essential task was to use it with musical systems for performances. Later with its widespread acceptance, the same protocol applies to the communication of data of any sort such as text, images, sound etc. (Figure 4).

The main computer performs Markov Chain operations in real time and creates a new content when a spectator presses a button. The representation of display interfaces and the OSC network communication were developed in the Processing Development Environment. ${ }^{13}$

Figure 4. Text Generation Algorithm

\begin{tabular}{|c|c|c|}
\hline $\begin{array}{l}\text { Random Title Selection } \\
\text { from an Array of Titles } \\
\text { EALANCE } \\
\text { COMPOSE } \\
\text { DERACEED } \\
\text { EROURANT } \\
\text { EROUANG } \\
\text { ESOERK } \\
\text { MODOAAE SCC }\end{array}$ & $\begin{array}{l}\text { Text Generation with } \\
\text { Markov Operations } \\
\text { Markonify Python Library } \\
\text { String Replacement }\end{array}$ & $\begin{array}{l}\text { Generated Statements presented } \\
\text { on a display } \\
\text { Separated Letters sent to } \\
\text { Slave Units via OSC Protocol }\end{array}$ \\
\hline
\end{tabular}

Markovify is a simple Markov chain generator that generates random sentences from a given text. According to Singer-Vine, Markovify works

12. Matthew Wright, Open Sound Control: an enabling technology for musical networking. Organised Sound 10, no. 3 (2005).

13. Casey Reas, and Ben Fry, Processing: a programming handbook for visual designers and artists (Cambridge, Massachusetts: The MIT Press, 2007). 
efficiently with considerably large, well-punctuated contents. ${ }^{14}$ Consequently, Being and Time text was organized with necessary punctuation structuring that is required by the Markovify Library (Figure 5).

Figure 5. Data Flow Configuration

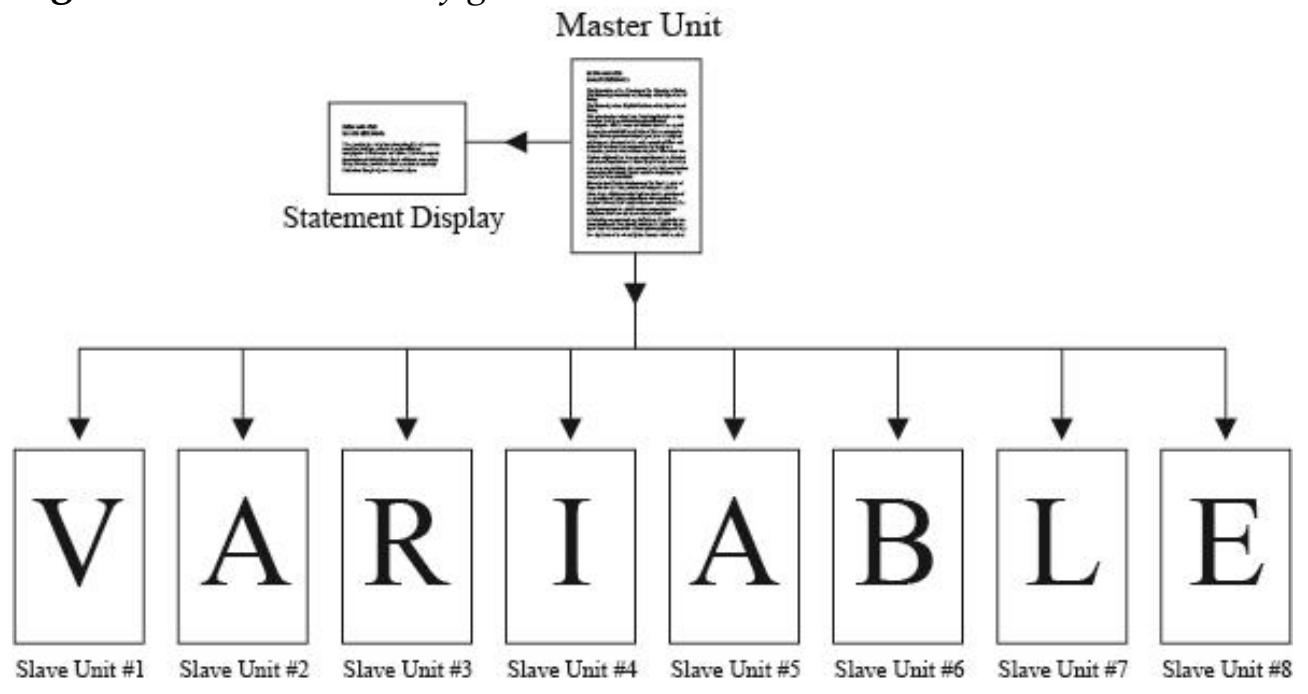

The main structure of the installation is built on a facade of a stainless reflective steel with dimensions of $60 \mathrm{~cm}$ to $170 \mathrm{~cm}$. With its minimalist view, its facade serves as a mirror reflecting its gallery space and all its sophisticated technology have been hidden at the behind. Thus, visitors to the exhibition are invited to become an immersive element for the artwork with their reflected images on the reflective surface.

As seen in Figure 6, very slim white fluorescent lights have been installed in between the LCD screens as solid separators. The aesthetical decision made for the choice of fluorescents aims to enhance the feeling of contrast between the artwork' tactile materiality and digital non-materiality. In addition to the main structure, a smaller LCD display was placed in a black housing and it was positioned to the right hand side of the piece to display conventional information about the art piece. Participants were permitted to use the button on the box to generate new versions for the piece.

14. Retrieved from https://bit.ly/2xQFVnE. 
Figure 6. Variable (from an exhibition)

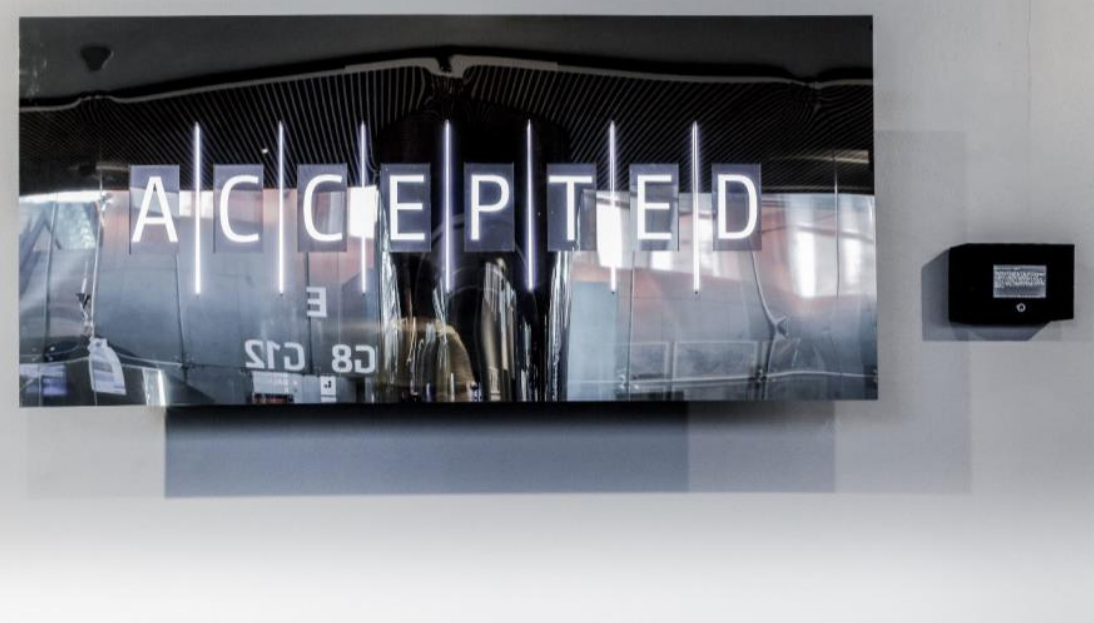

With the use of the Machine Learning algorithm, a new title and a statement is generated instantly as soon as a person interacts with the artpiece. Simultaneously, the generated title word is split into its letters and they are placed on each of the display screens separately accompanying a flowing text animation. Since the new generated texts are commonly contained with ontological questions based on the concept of "being," the frequent use of the words "being" are replaced by the generated artwork's titles with a string operation. Correspondingly, the artistic statement encapsulates a new ontological content that discusses on a new title for the artwork based on Heidegger' s philosophical point of view.

\section{Conclusion}

Inevitably technology has a great impact on shaping the way we perceive art with many intertwined dimensions. Needless to say that it is not an unexpected result since it has always been true for the cases in which we observe the inclusion of technology in the art-making process. Previously it could have been the invention of a new painting technique with the use of an emerging apparatus, today it is just another advancement in the technical implementation and most likely that it is based on concurrent digital technologies. Similarly, when machine learning became available for the artists to investigate, its creative potential has been intensively explored by the group of artists who are excited with the utilization of technology in the art-making process. This study aims to describe the methodologies behind the way in which the artwork "Variable" has 
been produced. In addition to all the research that has been studied in the field of machine intelligence, this artwork aspires to bring about an opportunity to discuss the notion of authenticity. As a result of the attempt to make art with the use of machine learning techniques, it is observed that we have limited information about human intelligence functions when we consider the boundaries of the creativity taking place in the arts.

\section{Bibliography}

Cho, Sung-Bae, and Hong-Hee Won. Machine learning in DNA microarray analysis for cancer classification. In Proceedings of the First Asia-Pacific bioinformatics conference on Bioinformatics 19 (2003), 189-198.

Cooper, G. F., C. F. Aliferis, R. Ambrosino, J. Aronis, B. G. Buchanan, R. Caruana, et al. An evaluation of machine-learning methods for predicting pneumonia mortality. Artificial Intelligence in Medicine 9, no. 2 (1997), 107-138.

Cruz, Joseph A., and David S. Wishart. Applications of machine learning in cancer prediction and prognosis. Cancer Informatics 2 (2006), 59-77.

Fiebrink, Rebecca. Machine Learning as Meta-Instrument: Human-Machine Partnerships Shaping Expressive Instrumental Creation. In Musical Instruments in the 21st Century, ed. Till Bovermann, Alberto de Campo, Hauke Egermann, Sarah-Indriyati Hardjowirogo, and Stefan Weinzierl, 137-151. Singapore: Springer, 2017.

Géron, Aurélien. Hands-On Machine Learning With Scikit-Learn and Tensor Flow: Concepts, Tools, and Techniques to Build Intelligent Systems. Sebastopol, CA: O’Reilly Media, 2017.

Mitchell, Tom M. Machine Learning. New York: McGraw-Hill, 1997.

Patel, Jigar, Sahil Shah, Priyank Thakkar, and K. Kotecha, Predicting stock market index using fusion of machine learning techniques. Expert Systems with Applications 42, no. 4 (2015), 2162-2172.

Pomerleau, D., and T. Jochem. Rapidly adapting machine vision for automated vehicle steering. IEEE Expert 11, no. 2 (1996), 19-27.

Reas, Casey, and Ben Fry. Processing: a programming handbook for visual designers and artists. Cambridge, Massachusetts: The MIT Press, 2007.

Samuel, Arthur L. Some studies in machine learning using the game of checkers. IBM Journal of Research and Development 3, no. 3 (1959), 210-229.

Schumaker, Robert P., and Hsinchun Chen. Textual analysis of stock market prediction using breaking financial news: The AZFin text system. ACM Transactions on Information Systems (TOIS) 27, no. 2 (2009).

Waibel, A., T. Hanazawa, G. Hinton, K. Shikano, and K. J. Lang. Phoneme recognition using time-delay neural networks. IEEE Transactions on Acoustics, Speech, and Signal Processing 37, no. 3, (1989), 328 - 339.

Wright, Matthew. Open Sound Control: an enabling technology for musical networking. Organised Sound 10, no. 3 (2005), 193-200.

Yoo, Paul D., Maria H. Kim, and Tony Jan. Machine learning techniques and use of event information for stock market prediction: A survey and evaluation. International 
Vol. 6, No. 3

Artut: Using a Machine Learning Algorithm to Create...

Conference on Computational Intelligence for Modeling, Control and Automation (CIMCA 2005), 835-841. Piscataway, NJ: IEEE, 2005. 